\title{
Gestational choriocarcinoma with uterine serosal metastasis mimicking ruptured ectopic pregnancy: A case report
}

\author{
YUKI SAKAMOTO, YUJI TAKEI, HIROYUKI FUJIWARA, \\ SHIZUO MACHIDA, AKIYO TANEICHI and MITSUAKI SUZUKI
}

Department of Obstetrics and Gynecology, Jichi Medical University, Tochigi 329-0498, Japan

Received June 8, 2014; Accepted February 18, 2015

DOI: 10.3892/ol.2015.2999

\begin{abstract}
Primary gestational choriocarcinoma is commonly present in the uterus in cases of atypical genital bleeding. Symptoms similar to those of an ectopic pregnancy develop when an extra-uterine lesion is present in the abdominal cavity, and lesions have been detected in the ovaries and fallopian tubes in a number of cases. In the present study, we describe a patient with choriocarcinoma that metastasized to the uterine serosa and caused symptoms similar to those of an ectopic pregnancy. The patient was a 30 -year-old female who presented to our hospital with atypical genital bleeding and a positive pregnancy test 3 months after missed abortion at 10 weeks of gestation. Transvaginal ultrasonography revealed the absence of a gestational sac in or outside the uterus, and intra-abdominal bleeding was noted. An ectopic pregnancy was suspected based on these findings, and emergency laparotomy was performed. A hemorrhagic mass was present on the uterine serosa, and was subsequently resected. Trophoblastic disease was suspected following histopathological examination, for which intra-uterine curettage was performed and choriocarcinoma was diagnosed. Lung metastasis was detected on computed tomography, and a high serum human chorionic gonadotropin (hCG) level persisted following surgery. The lesion disappeared following five cycles of methotrexate+ etoposide+actinomycin D therapy, which was performed as postoperative chemotherapy, and the patient's serum hCG level decreased to below the detection limit. In this case of choriocarcinoma, the primary lesion was present in the uterus and had metastasized to the uterine serosa, which is a very rare metastatic site. This uterine serosal metastatic lesion bled and caused symptoms similar to those of an ectopic pregnancy. Certain patients who undergo surgery for a suspected
\end{abstract}

Correspondence to: Dr Yuji Takei, Department of Obstetrics and Gynecology, Jichi Medical University, 3311-1 Yakushiji, Shimotsuke, Tochigi 329-0498, Japan

E-mail: ytakei@jichi.ac.jp

Key words: gestational choriocarcinoma, uterine serosal metastasis, ectopic pregnancy peritoneal pregnancy may have gestational choriocarcinoma, similar to this case.

\section{Introduction}

Choriocarcinoma is a malignant disease characterized by abnormal trophoblastic hyperplasia and anaplasia, absence of chorionic villi, hemorrhage, and necrosis. Approximately $25 \%$ of choriocarcinoma cases follow abortion or tubal pregnancy, $25 \%$ are associated with term or preterm gestation, and the remaining $50 \%$ arise from hydatidiform moles, however, few hydatidiform moles are estimated to progress to choriocarcinoma. Primary gestational choriocarcinoma is identified in the uterus in cases of atypical genital bleeding; however, gestational choriocarcinoma that primarily occurs outside the uterus is rare. Primary lesions have previously been detected in the ovary, fallopian tube, omentum, lung and liver (1-5). Choriocarcinoma may present with various symptoms depending on the disease site. For example, pulmonary lesions may induce cough and hemoptysis, whereas brain lesions may induce headaches and visual impairment. Clinical symptoms similar to those of an ectopic pregnancy, including abdominal pain and intra-abdominal bleeding, may develop when a primary lesion is present in the abdominal cavity outside the uterus $(1,2,4,5)$. Conversely, the lung, liver, brain, vagina and digestive tract are frequently metastasized by gestational choriocarcinoma that primarily developed in the uterus (6). In the current patient, the primary lesion was present in the uterus and metastasized to the uterine serosa, which is a very rare metastatic site. To the best of our knowledge, this is the first study to describe gestational choriocarcinoma with uterine serosal metastasis. Written informed consent was obtained from the patient.

\section{Case report}

The patient was a 30-year-old female, gravida 2 and para 1 . Her previous and familial medical histories were unremarkable. The patient was diagnosed with missed abortion and treated with intra-uterine curettage at 10 weeks of gestation by a physician. Fetal components were macroscopically observed in the uterine content, and no hydatidiform mole was present. A small volume of genital bleeding had persisted intermittently.

The patient presented to the Department of Obstetrics and Gynecology at Jichi Medical University, Japan for the first 


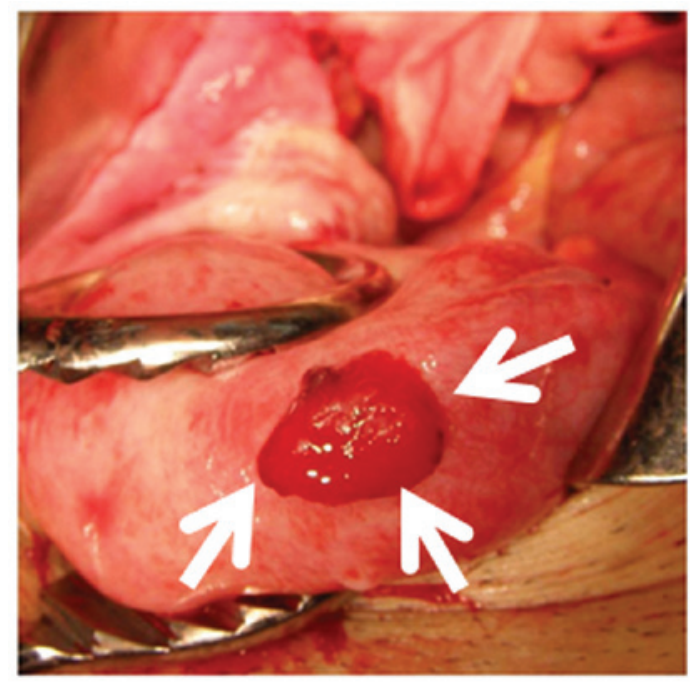

Figure 1. Macroscopic findings of the uterine serosal metastatic lesion. A 20x10-mm mass with oozing blood protruded from the serous surface in the uterine fundus on the left side (arrow).

time three months after intra-uterine curettage with a positive result on a commercial pregnancy test and abdominal pain. Based on a pelvic examination and transvaginal ultrasonography, missed abortion in early pregnancy was considered, as a gestational sac and fetus were not detected. Since her abdominal pain was very mild, a closer observation was selected. No ultrasound findings suggestive of gestational trophoblastic neoplasia (GTN) were noted at that time. The patient re-visited our department two days later as her abdominal pain and bleeding had exacerbated. Transvaginal ultrasonography revealed that no gestational sac was present in the uterus, and blood clots were retained in the vesico-uterine and the pouch of Douglas, for which emergency surgery was performed on the same day due to a suspected ruptured ectopic pregnancy. The patient's serum human chorionic gonadotropin (hCG) level was $12,000 \mathrm{mIU} / \mathrm{ml}$. Laparotomy revealed a large blood clot in the pouch of Douglas. Intra-abdominal blood loss was $550 \mathrm{ml}$. A 20x10-mm mass with oozing blood protruded from the surface in the uterine fundus (Fig. 1), and its mass was judged to be the site of the peritoneal pregnancy. The mass and adjacent myometrium were resected en bloc. The patient's bilateral fallopian tubes and ovaries were normal, and her postoperative course was favorable.

However, on postoperative day 6 the patient's serum hCG level was $10,000 \mathrm{mIU} / \mathrm{ml}$, demonstrating unfavorable reduction, and no villous architecture or fetal component was noted in the excised specimen, whereas trophoblast proliferation was observed in the uterine tissue on histopathological examination (Fig. 2). A trophoblastic disease, such as choriocarcinoma and hydatidiform mole, was suspected; therefore, intra-uterine curettage, head and thoracoabdominal computed tomography (CT), and pelvic magnetic resonance imaging (MRI) were performed. A histopathological examination of the endometrial curettage sample revealed the absence of villous tissue, whereas the proliferation of prominently atypical syncytiotrophoblasts and cytotrophoblasts was noted, and a number of cells were mitotic (Fig. 3). The patient was diagnosed with choriocarcinoma based on these findings. The histopathological results of
A

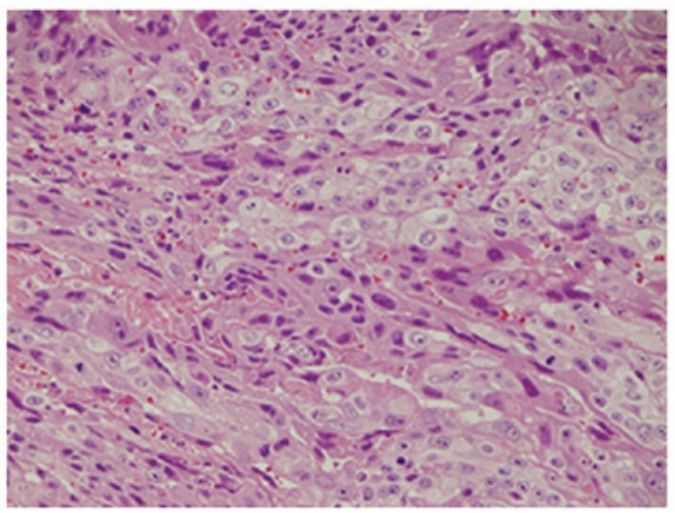

B

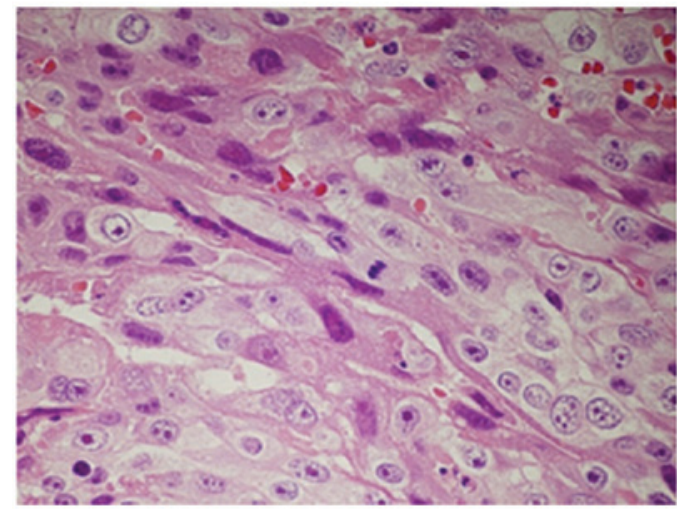

Figure 2. Histopathological findings of the uterine serosal metastatic lesion. The proliferation of syncytiotrophoblasts and cytotrophoblasts was observed in the uterine tissue. Hematoxylin and eosin staining. Magnification, (A) $\mathrm{x} 200$; (B) $\mathrm{x} 400$.

A

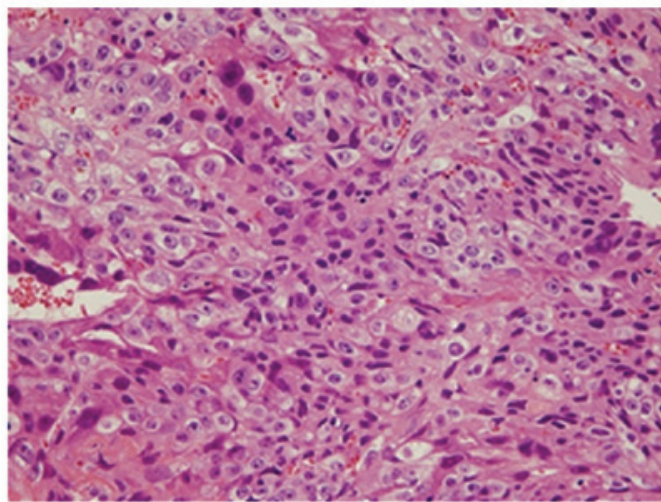

B

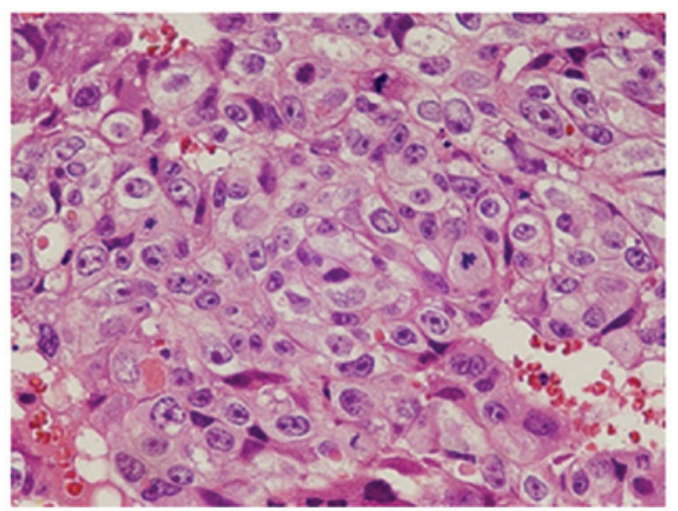

Figure 3. Histopathological findings of intra-uterine curettage. The proliferation of prominently atypic syncytiotrophoblasts and cytotrophoblasts was observed. Hematoxylin and eosin staining. Magnification, (A) x200; (B) $\times 400$ 


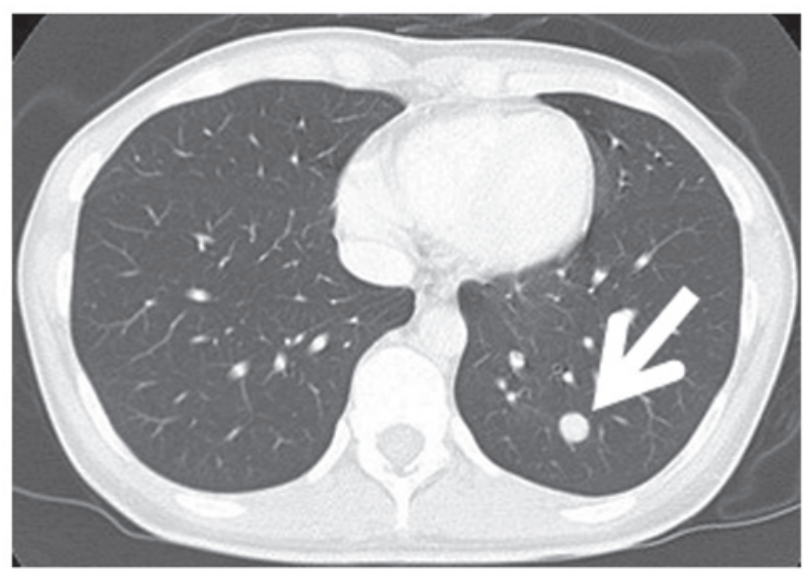

Figure 4. Computed tomography findings. A 9-mm round nodule was present in the left lower lung field (arrow).

the resected uterine lesion and endometrial curettage sample revealed that the primary lesion was present in the uterus and metastasized to the uterine serosa. A 9-mm round nodule was present in the left lower lung field on thoracoabdominal CT (Fig. 4), and a total of six small nodules were scattered in the bilateral lung fields. No abnormalities were observed in the abdominal region. Head CT and pelvic MRI also revealed no abnormalities.

For this patient, the FIGO score was 5 [age, 30 years; antecedent pregnancy, missed abortion; interval from index pregnancy, <4 months; pre-treatment serum hCG, 12,000 IU/1; largest tumor size, $2 \mathrm{~cm}$; site of metastasis, lung and uterine serosa; number of metastases, 8 ( 7 in the lung and 1 in the uterine serosa); previously failed chemotherapy, none], and the FIGO stage was III. Although it was a low-risk GTN, choriocarcinoma was diagnosed based on the histopathological findings. Accordingly, it was considered that this case should be treated as high-risk GTN, and five cycles of methotrexate+ etoposide+actinomycin D (MEA) therapy were administered.

Endometrial cytology using Softsite ${ }^{\circledR}$ (Softmedical, Tokyo, Japan) became negative following two cycles of MEA therapy. Although endometrial cytology is not a routine assessment for GTN under treatment, we performed it to confirm the disappearance of choriocarcinoma cells. The patient's serum hCG level decreased below the detection limit after three cycles. The complete disappearance of the metastatic lesion in the lung was confirmed by CT after five cycles. No recurrence has occurred four years and 9 months after the completion of MEA therapy. In addition, the patient became pregnant and gave birth one year and 3 months after the completion of MEA therapy.

\section{Discussion}

The findings of this case indicated that gestational choriocarcinoma may cause uterine serosal metastasis. Furthermore, certain patients who undergo surgery for a suspected peritoneal pregnancy may have gestational choriocarcinoma, similar to this case.

Gestational choriocarcinoma may cause uterine serosal metastasis. To the best of our knowledge, this is the first case study in which the primary lesion was present in the uterus and metastasized to the uterine serosa. A PubMed search was conducted using two keywords, 'choriocarcinoma' and 'serosal metastasis' or 'peritoneal metastasis'. No case report was identified with regard to metastatic choriocarcinoma from a primary lesion in the uterus to the uterine serosa. A similar case report describing gestational choriocarcinoma on the surface of subserosal leiomyoma was identified (7). However, this study was different from ours since the primary lesion was present on the surface of subserosal leiomyoma. In our case, a metastatic lesion, but not a primary lesion, was present in the uterine serosa.

The lungs, liver, brain, vagina and digestive tract are common sites of metastasis (6). Three mechanisms have been proposed for uterine serosal metastasis. Firstly, the lesion may have been hematogenously implanted on the uterine serosa. Lung metastasis was also present in this patient, which suggested hematogenous metastasis. It remains unclear whether the lesion returned to the uterine serosa after entering the systemic circulation or whether it directly reached the uterine serosa through a myometrial blood vessel. Secondly, the intra-uterine lesion may have passed through the fallopian tubes, dropped into the abdominal cavity, and engrafted on the uterine serosa. For example, in a peritoneal pregnancy, a fertilized egg may fall out of the fallopian tube into the abdominal cavity and engraft on the peritoneum. Thirdly, the intra-uterine lesion may have penetrated the myometrium and reached the uterine serosa. The possibility of intra-abdominal bleeding caused by penetration of the myometrium by GTN and an actual case of myometrial penetration by a lesion have been reported previously (8). However, this could not be pathologically investigated in our patient since the uterus was not excised. Therefore, we cannot confirm which hypothesis was the most relevant. However, the first hypothesis appears to be the most plausible. We suspect that the lesion directly reached the uterine serosa through a myometrial blood vessel. The second and third hypotheses are less plausible than the first for the following reasons. According to the second hypothesis, an intra-uterine lesion detaching from the fallopian tube is more likely to form a lesion in the pouch of Douglas than on the surface of the uterine serosa. This is due to the fact that a detached lesion is more likely to go deeper into the abdomen. According to the third hypothesis, a lesion penetrating the myometrium is more likely to be detected in the myometrium by ultrasonography or MRI. However, in the present case, no lesion was detected in the myometrium by ultrasonography or MRI.

Certain patients who undergo surgery for suspected peritoneal pregnancy may have gestational choriocarcinoma, similar to this case. When a uterine serosal metastatic lesion bleeds, symptoms similar to those of an ectopic pregnancy develop. Gestational choriocarcinoma arising from the ovary, fallopian tube, omentum, liver and the surface of the subserosal myoma have been reported previously, and all of these were diagnosed as choriocarcinoma by postoperative histopathological examinations following surgery performed for a suspected ectopic pregnancy $(1,2,4,5,7)$. Symptoms similar to those of an ectopic pregnancy develop when the lesion bleeds, regardless of whether the intra-abdominal lesion is a primary or metastatic lesion. When the lesion is surgically resected, a macroscopic examination is required to determine whether chorionic tissue is present in the lesion. In addition, it is essential to ensure 
that the lesion is an ectopic pregnancy by histopathological examination and to confirm postoperative reduction in the serum hCG level to below the detection limit. If these attempts fail, the diagnosis of choriocarcinoma may be delayed. This case highlights the significance of these confirmations.

The findings of the present case indicated that gestational choriocarcinoma may cause uterine serosal metastasis. Furthermore, certain patients who undergo surgery for suspected peritoneal pregnancy may have gestational choriocarcinoma, similar to this case. Therefore, it is essential to confirm the postoperative histopathological diagnosis and reduction in serum hCG level in patients who have undergone surgery for a peritoneal pregnancy, such as this patient.

\section{References}

1. Wan X, Li J and Xie X: Extrauterine choriocarcinoma of the greater omentum after tubal pregnancy: case report. Int $\mathrm{J}$ Gynecol Cancer 16: 1476-1478, 2006.
2. Mittal S, Aird I and Haugk B: Gestational choriocarcinoma in liver mimicking ruptured ectopic pregnancy. J Obstet Gynaecol 32: 499, 2012

3. Maestá I, Leite FV, Michelin OC and Rogatto SR: Primary pulmonary choriocarcinoma after human chorionic gonadotropin normalization following hydatidiform mole: a report of two cases. J Reprod Med 55: 311-316, 2010.

4. Sakumoto K, Nagai Y, Inamine M and Kanazawa K: Primary omental gestational choriocarcinoma ascertained by deoxyribonucleic acid polymorphism analysis. Gynecol Oncol 97: 243-245, 2005.

5. Küçüközkan T, Savan K, Aydin E, Sönmez S, Duran B and Kaygi O: Choriocarcinoma associated with ectopic pregnancy after tubal sterilisation. Acta Obstet Gynecol Scand 71: 636- 638, 1992.

6. Berkowitz RS and Goldstein DP: Chorionic tumors. N Eng J Med 335: 1740-1748, 1996.

7. Chen MJ, Yang JH, Lin MC, Ho HN and Yang YS: An unusual gestational choriocarcinoma occurring primarily on the surface of a subserous leiomyoma. BJOG 111: 188-190, 2004.

8. Ashraf-Ganjooei T and Ghaemmaghami F: Patients with presenting unusual manifestations with gestational trophoblastic neoplasm: case series and review of literatures. Arch Gynecol Obstet 277: 465-470, 2008. 